\title{
Behavioral characterization of mice overexpressing human dysbindin-1
}

\author{
Norihito Shintani ${ }^{1 \dagger}$, Yusuke Onaka ${ }^{1 \dagger}$, Ryota Hashimoto ${ }^{2,3^{*+}}$, Hironori Takamura ${ }^{2 \dagger}$, Tsuyoshi Nagata $^{1 \dagger}$, \\ Satomi Umeda-Yano ${ }^{4}$, Akihiro Mouri ${ }^{5}$, Takayoshi Mamiya ${ }^{5}$, Ryota Haba', Shinsuke Matsuzaki ${ }^{2,6,7}$, Taiichi Katayama ${ }^{6}$, \\ Hidenaga Yamamori ${ }^{2,4}$, Takanobu Nakazawa ${ }^{8}$, Kazuki Nagayasu ${ }^{8}$, Yukio Ago ${ }^{9}$, Yuki Yagasaki ${ }^{10}$, Toshitaka Nabeshima $^{11}$, \\ Masatoshi Takeda ${ }^{2,3}$ and Hitoshi Hashimoto ${ }^{1,2,8}$
}

\begin{abstract}
Background: The dysbindin-1 gene (DTNBP1: dystrobrevin binding protein 1) is a promising schizophrenia susceptibility gene, known to localize almost exclusively to neurons in the brain, and participates in the regulation of neurotransmitter release, membrane-surface receptor expression, and synaptic plasticity. Sandy mice, with spontaneous Dtnbp1 deletion, display behavioral abnormalities relevant to symptoms of schizophrenia. However, it remains unknown if dysbindin-1 gain-of-function is beneficial or detrimental.

Results: To answer this question and gain further insight into the pathophysiology and therapeutic potential of dysbindin-1, we developed transgenic mice expressing human DTNBP1 (Dys1A-Tg) and analyzed their behavioral phenotypes. Dys1A-Tg mice were born viable in the expected Mendelian ratios, apparently normal and fertile. Primary screening of behavior and function showed a marginal change in limb grasping in Dys1A-Tg mice. In addition, Dys 1A-Tg mice exhibited increased hyperlocomotion after methamphetamine injection. Transcriptomic analysis identified several up- and down-regulated genes, including the immediate-early genes Arc and Egr2, in the prefrontal cortex of Dys1A-Tg mice.

Conclusions: The present findings in Dys 1A-Tg mice support the role of dysbindin-1 in psychiatric disorders. The fact that either overexpression (Dys1A-Tg) or underexpression (Sandy) of dysbindin-1 leads to behavioral alterations in mice highlights the functional importance of dysbindin-1 in vivo.
\end{abstract}

Keywords: Dysbindin, DTNBP1, Dystrobrevin binding protein 1, Psychiatric disorder, Schizophrenia, Transgenic mice, Behavior, Methamphetamine, Phencyclidine, Immediate-early gene

\section{Background}

Dysbindin-1 (dystrobrevin binding protein 1) is an evolutionary conserved $40-\mathrm{kDa}$ coiled-coil-containing protein that binds to dystrobrevin and localizes exclusively to neurons in the brain [1]. Dysbindin-1 has been shown to participate in biogenesis of lysosome-related organelles complex 1 , which regulates trafficking to lysosomerelated organelles [2], regulation of neurotransmitter

\footnotetext{
* Correspondence: hashimor@psy.med.osaka-u.ac.jp

${ }^{\dagger}$ Equal contributors

${ }^{2}$ Molecular Research Center for Children's Mental Development, United

Graduate School of Child Development, Osaka University, Kanazawa

University, Hamamatsu University School of Medicine, Chiba University and

University of Fukui, 2-2 Yamadaoka, Suita, Osaka 565-0871, Japan

${ }^{3}$ Department of Psychiatry, Graduate School of Medicine, Osaka University,

2-2 Yamadaoka, Suita, Osaka 565-0871, Japan

Full list of author information is available at the end of the article
}

release [3-5], membrane surface expression of glutamate NMDA [6] and dopamine D2 $[7,8]$ receptors, and synaptic plasticity $[6,9]$.

Genetic variations in the human dysbindin-1 gene (DTNBP1) have been shown to be associated with schizophrenia [5,10], bipolar disorder [11], and methamphetamine (METH) psychosis [12], as well as neurocognitive functions in healthy subjects $[13,14]$. In postmortem brain from schizophrenic patients, decreased dysbindin-1 expression has been demonstrated in the prefrontal cortex [15], cerebral cortex [16], and intrinsic glutamatergic terminals of the hippocampal formation [17].

Sandy mice completely lack dysbindin-1 protein because of spontaneous deletion of introns 5-7 of the Dtnbp1 gene in DBA/2 J mice [2]. These mice display a 
variety of behavioral abnormalities relevant to symptoms of schizophrenia, including hypoactivity, heightened anxiety-like responses, reduced social interaction [18], deficits in both long-term [19] and working memory [20], and impairments in contextual fear conditioning [9]. As potential mechanisms for these behavioral abnormalities, Sandy mice have been shown to exhibit reduced dopamine transmission in the forebrain [18] and destabilization of snapin, which binds to SNAP25 and regulates calcium-dependent exocytosis [19].

The sandy mutation was backcrossed onto a C57BL/ $6 \mathrm{~J}$ background for at least 11 generations to obtain sdy/ B6 mice [21]. These mice show schizophrenia-like behaviors including hyperactivity, spatial learning and memory deficits, impaired working memory under challenging conditions, and disruption of dopamine/D2-related mechanisms that regulate cortical function and neuronal excitability [21,22]. sdy/B6 mice also exhibit increased impulsive and compulsive behaviors relevant to psychiatric disorders [23].

Thus, a growing body of evidence implicates dysbindin1 in psychiatric disorders. However, because of failure to replicate genetic association studies [24], a lack of causal variants with a notable impact on disease risk that might contribute to schizophrenia [25], and methodological difficulties in postmortem brain research due to heterogeneity of tissues with respect to biochemical parameters, lifetime history of medications and physiological status at the time of death [26], it remains unclear how dysbindin-1 functions as a susceptibility gene for these disorders.

A recent study in mice and humans demonstrated an epistatic interaction between catechol-O-methyl transferase (COMT) and dysbindin-1 that modulates prefrontal function, specifically, subjects with reduced function of either COMT or dysbindin-1 show superb physiological performance, whereas those with reductions in both proteins have performance deficits [27]

As dysbindin-1 has both beneficial and detrimental effects in prefrontal cortical function, we performed a gainof-function study of dysbindin-1 by developing transgenic mice that express the human dysbindin-1 gene (Dys1A$\mathrm{Tg}$ ) and we analyzed their behavioral phenotypes.

\section{Results}

\section{Generation of Dys1A-Tg mice}

Dysbindin-1 exists as multiple isoforms produced by alternative mRNA splicing. Of these isoforms, dysbindin$1 \mathrm{~A},-1 \mathrm{~B}$, and $-1 \mathrm{C}$ are commonly expressed transcripts, although dysbindin-1B is not expressed in mice [17]. Here, we constructed a transgene expressing human dysbindin-1A isoform cDNA, C-terminally fused to GFP (hDTNBP1-GFP) [28] under control of the CA promoter, consisting of a modified promoter of the chicken gene for $\beta$-actin with a cytomegalovirus immediate-early enhancer [29] (Figure 1A). Pronuclear injection of the transgene into fertilized C57BL/6 J mouse eggs was performed, generating Dys1A-Tg mice expressing human dysbindin-1 protein (Figure 1B). There was no apparent compensatory decrease in endogenous (mouse) dysbindin1 expression in Dys1A-Tg mice (Figure 1C). This indicated that total expression levels of dysbindin-1 are significantly increased in Dys1A-Tg mice. Since it was revealed that line 1 Dys1A-Tg mice express higher levels of human dysbindin-1, we performed the following experiments in this line. Through subsequent matings with wild-type mice, offspring were obtained at a frequency not significantly different from the expected Mendelian ratio (Dys1A-Tg, 47.4\%; $n=190 ; P=0.157$ by $\chi^{2}$ analysis). Dys1A-Tg mice were fertile, and germline transmission of the transgene was confirmed for at least 10 generations, with an apparently normal coat color (data not shown). Reverse transcription-polymerase chain reaction (RT-PCR) analysis detected $h D T N B P 1-G F P$ transcript expression in various tissues of Dys1A-Tg mice, but not in wild-type mice (Figure 1D). Quantitative real-time RT-PCR revealed that $h D T N B P 1-G F P$ mRNA is expressed in the brain cortex in Dys1A-Tg (line 1) mice but not in wild-type mice, whereas mouse Dtnbp1 mRNA is similarly expressed in the brain cortex in Dys1A-Tg and wild-type mice (hDTNBP1-GFP mRNA, Dys1A-Tg, $3.77 \pm 0.66, \mathrm{n}=5$; wild-type, $0.00 \pm 0.00, \mathrm{n}=4, \quad P=0.001 ;$ mouse Dtnbp 1 mRNA, Dys1A-Tg, $0.91 \pm 0.06, \mathrm{n}=5$; wild-type, $1.00 \pm$ $0.09, \mathrm{n}=4, \quad P=0.42$; normalized to glyceraldehyde-3phosphate dehydrogenase (GAPDH) mRNA and relative to mouse Dtnbp1 mRNA in wild-type mice).

\section{Behavioral characterization of Dys $1 \mathrm{~A}-\mathrm{Tg}$ mice under basal conditions}

To determine if dysbindin-1 overexpression affects physical and behavioral profiles in mice, we used the standardized SHIRPA (SmithKline Beecham, Harwell, Imperial College, Royal London Hospital, phenotype assessment) screening program [30], which was slightly modified as described [31]. Of the 28 components in the primary SHIRPA screening, a significant difference between Dys1A$\mathrm{Tg}$ and wild-type littermates was observed only in limb grasping behavior $(P=0.045$, Mann-Whitney $U$ test; Table 1). Moreover, $\chi^{2}$ analysis revealed a significant genotype difference in the number of mice that showed limb grasping behavior (Dys1A-Tg, 72\%, $\mathrm{n}=18$; wild-type, 38\%, $\left.\mathrm{n}=16 ; \chi^{2}=10.7, \mathrm{P}=0.0011\right)$. There were no appreciable differences in the other motor functions, appearance, sensory functions, or anxiety levels between Dys1A-Tg and wild-type mice (Table 1). Further behavioral analyses in secondary screening showed no genotype differences in distance traveled, rearing, and time spent in the center region of the open-field test (Figure 2A-C), pre-pulse inhibition (PPI) levels (Figure 2D), startle responses to main 


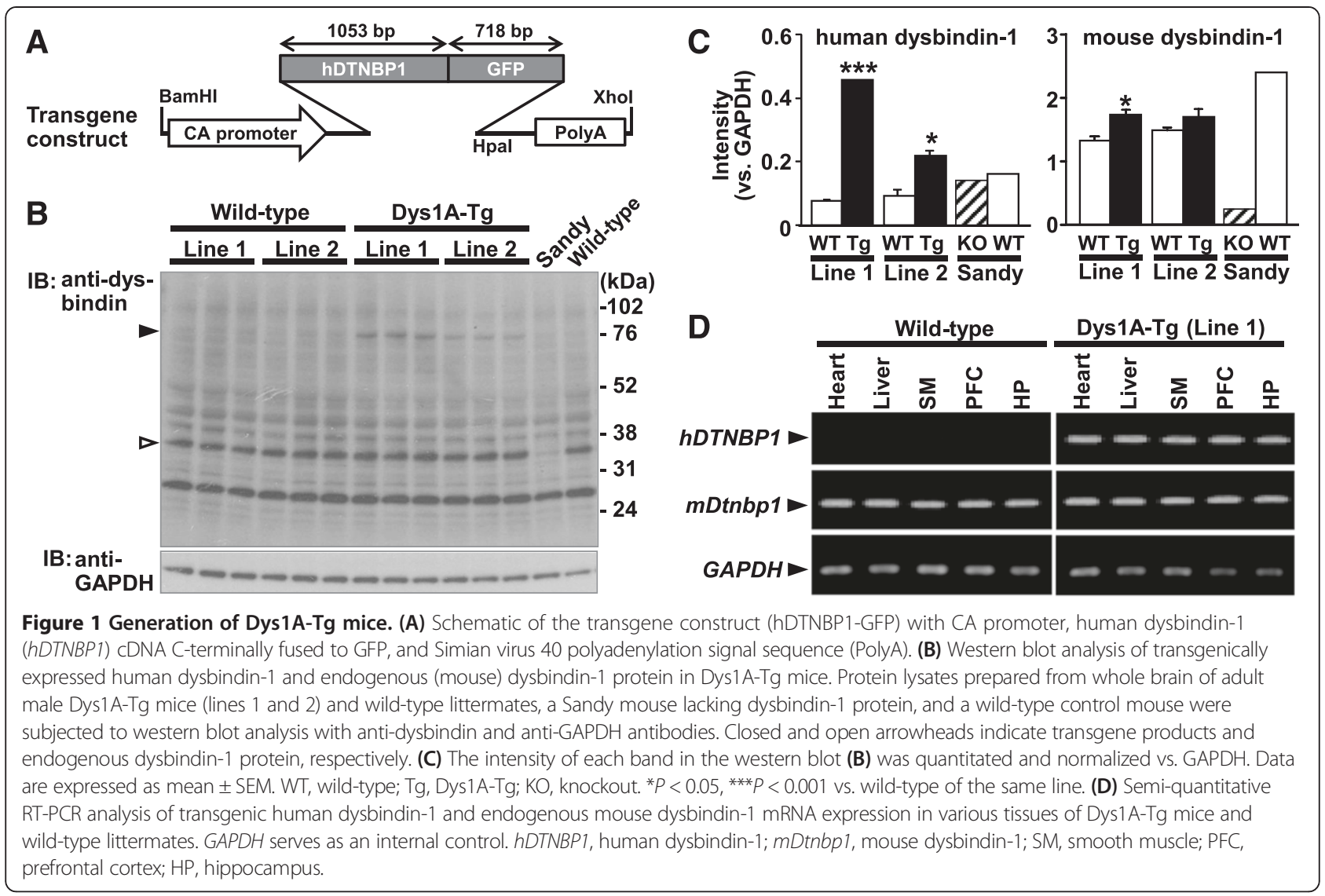

pulses of $120 \mathrm{~dB}$ (Dys1A-Tg, $407 \pm 53$; wild-type: $395 \pm 66$; arbitrary unit), and latency to fall over in three sessions of the accelerated rotarod test (Figure 2E). These results suggest that under basal conditions, Dys1A-Tg mice have no gross behavioral abnormalities except in limb grasping behavior.

\section{Behavioral response to acute treatment with METH and phencyclidine in Dys1 A-Tg mice}

Mice administered with psychostimulants (e.g., METH) and non-competitive N-methyl-D-aspartate receptor antagonists (e.g., phencyclidine, PCP) serve as animal models for psychiatric disorders including schizophrenia $[32,33]$. DTNBP1 has been implicated as a risk factor for psychiatric disorders such as schizophrenia [9-11,13-20], therefore we examined behavioral responses to acute treatment with METH and PCP in Dys1A-Tg mice. In the open-field test, repeated two-way analysis of variance (ANOVA) revealed a significant time and genotype interaction in METH-induced hyperlocomotion at $1 \mathrm{mg} / \mathrm{kg}$ $\left(F_{11,572}=2.28, P=0.010\right)$, while $\mathrm{METH}$ at $2 \mathrm{mg} / \mathrm{kg}$ had no significant effect on the interaction $\left(F_{11,374}=0.784\right.$, $P=0.66$; Figure 3A). Dys1A-Tg mice administered with $1 \mathrm{mg} / \mathrm{kg}$ METH showed significantly increased total locomotor activity for $30 \mathrm{~min}$ immediately after METH administration compared with wild-type mice $(P<0.05$; Figure 3A right). After METH administration, PPI was significantly disrupted (treatment effect, $F_{2}, 272=6.75, P=$ $0.0016)$ but no significant genotype effect was identified $\left(F_{1}, \quad 272=1.27, \quad P=0.26\right.$; repeated three-way ANOVA; Figure $3 \mathrm{~B})$. Two-way ANOVA revealed a significant effect of PCP on locomotor activity $\left(F_{2,25}=16.6, P<0.0001\right)$ but not genotype effect $\left(F_{1,25}=0.005, P=0.94\right.$; two-way ANOVA; Figure $3 \mathrm{C}$ ). The novel object investigation test was subsequently performed using mice that received PCP (Figure 3D). In this test, exploratory behavior towards a novel object is assessed. Two-way ANOVA revealed a significant effect of PCP $\left(F_{2,19}=7.74, P=0.0035\right)$ but not the interaction of PCP and genotype $\left(F_{2,19}=0.718, P=0.50\right)$.

\section{Behavioral response to chronic PCP treatment in Dys1 A-Tg} mice

We also examined behavioral responses to chronic treatment with PCP for 14 days in Dys1A-Tg mice. To reduce the number of animals, mice were subjected to a battery of behavioral tests (Figure 4A). Dys1A-Tg and wild-type littermates $(n=12-14)$ were subcutaneously injected with PCP (3 or $10 \mathrm{mg} / \mathrm{kg}$ ) or vehicle (saline) once a day for 14 days, as previously reported [34]. PCPinduced increases in locomotion were not different 
Table 1 SHIRPA primary screening in Dys1A-Tg mice

\begin{tabular}{|c|c|c|c|}
\hline Paradigm and examination & Wild-type & Dys1A-Tg & $P$ value \\
\hline \multicolumn{4}{|l|}{ Appearance } \\
\hline Body weight (g) & $23.4 \pm 0.43$ & $23.1 \pm 0.40$ & 0.88 \\
\hline Body position & $4.00 \pm 000$ & $4.00 \pm 0.00$ & N.D. \\
\hline Respiration rate & $2.00 \pm 0.00$ & $2.06 \pm 0.06$ & 0.35 \\
\hline Heart rate & $1.06 \pm 0.06$ & $1.11 \pm 0.08$ & 0.62 \\
\hline Tremor & $0.13 \pm 0.09$ & $0.11 \pm 0.08$ & 0.90 \\
\hline Palpebral closure & $0.00 \pm 0.00$ & $0.00 \pm 0.00$ & N.D. \\
\hline Piloerection & $0.00 \pm 0.00$ & $0.00 \pm 0.00$ & N.D. \\
\hline Lacrimation & $0.00 \pm 0.00$ & $0.00 \pm 0.00$ & N.D. \\
\hline \multicolumn{4}{|l|}{ Motor function } \\
\hline Spontaneous activity & $3.00 \pm 0.00$ & $3.00 \pm 0.00$ & N.D. \\
\hline Transfer arousal & $3.94 \pm 0.17$ & $4.00 \pm 0.16$ & 0.79 \\
\hline Gait & $0.00 \pm 0.00$ & $0.03 \pm 0.03$ & 0.35 \\
\hline Pelvic elevation & $2.06 \pm 0.06$ & $2.06 \pm 0.06$ & 0.93 \\
\hline Tail elevation & $1.81 \pm 0.10$ & $1.83 \pm 0.09$ & 0.88 \\
\hline Trunk curl & $0.38 \pm 0.13$ & $0.44 \pm 0.12$ & 0.69 \\
\hline Limb grasping & $0.38 \pm 0.13$ & $0.72 \pm 0.11$ & 0.045 \\
\hline Grip strength & $2.56 \pm 0.13$ & $2.72 \pm 0.14$ & 0.44 \\
\hline Righting reflex & $0.00 \pm 0.00$ & $0.00 \pm 0.00$ & N.D. \\
\hline Contact reflex & $1.00 \pm 0.00$ & $0.97 \pm 0.03$ & 0.35 \\
\hline Negative geotaxis & $0.00 \pm 0.00$ & $0.00 \pm 0.00$ & N.D. \\
\hline \multicolumn{4}{|l|}{ Sensory function } \\
\hline Touch escape & $1.81 \pm 0.10$ & $1.89 \pm 0.11$ & 0.64 \\
\hline Positional passivity & $0.03 \pm 0.03$ & $0.06 \pm 0.06$ & 0.97 \\
\hline Visual placing & $1.88 \pm 0.09$ & $1.94 \pm 0.06$ & 0.48 \\
\hline Corneal reflex & $1.00 \pm 0.00$ & $1.00 \pm 0.00$ & N.D. \\
\hline Toe pinch & $2.75 \pm 0.11$ & $2.72 \pm 0.11$ & 0.86 \\
\hline \multicolumn{4}{|l|}{ Anxiety level } \\
\hline Urination & $0.38 \pm 0.13$ & $0.17 \pm 0.09$ & 0.18 \\
\hline Defecation & $2.56 \pm 0.49$ & $3.00 \pm 0.58$ & 0.75 \\
\hline Vocalization & $0.88 \pm 0.09$ & $0.89 \pm 0.08$ & 0.90 \\
\hline Provoked biting & $0.88 \pm 0.09$ & $0.83 \pm 0.09$ & 0.74 \\
\hline
\end{tabular}

Data are expressed as mean \pm SEM (Dys1A-Tg, $n=18$; wild-type, $n=16$ ). $P$ values were calculated using the Mann-Whitney $U$ test. Bold type indicates $P<0.05$. N.D., not different.

between Dys1A-Tg and wild-type mice (Figure 4B). In the FST, two-way ANOVA revealed a significant effect of PCP $\left(F_{2}, 70=12.5, P<0.0001\right)$ but not interaction between $\mathrm{PCP}$ and genotype $\left(F_{2,70}=0.848, P=0.43\right.$; Figure $\left.4 \mathrm{C}\right)$. As several mice injected with PCP at $10 \mathrm{mg} / \mathrm{kg}$ showed abnormal behavior such as increased locomotion in home cages, we subsequently performed the social interaction and novel object recognition tests in mice injected with $3 \mathrm{mg} / \mathrm{kg}$ PCP or vehicle only. In wild-type mice, as expected, PCP (3 mg/kg) significantly impaired social interaction and preference index in the novel object recognition test (Figure 4D, E). In contrast, Dys1A-Tg mice showed mildly attenuated responses to chronically administered PCP, but these did not reach statistically significant levels except for the social interaction test. In the social interaction test (Figure 4D), two-way ANOVA revealed no significant effect of $\mathrm{PCP}\left(F_{1,48}=1.28, P=\right.$ 0.26 ) but identified a significant interaction between PCP and genotype $\left(F_{1,48}=4.24, P=0.045\right)$. The post-hoc Tukey-Kramer test showed that chronic PCP reduces social interaction in wild-type mice only $(P<0.05)$, and there is a significant difference between Dys1A-Tg and wild-type mice injected with vehicle $(P<0.05)$. In the novel object recognition test, mice first freely explore two objects (training session), and memory retention for the objects evaluated after $24 \mathrm{~h}$ (test session). As statistical analysis showed no significant differences in training session behavior (data not shown), test session results are shown (Figure 4E). Two-way ANOVA revealed a significant PCP effect for the preference index $\left(F_{1,48}=11.8\right.$, $P=0.0012)$ but not interaction between PCP and genotype $\left(F_{1,48}=1.58, \mathrm{P}=0.22\right)$.

\section{Altered gene expression in Dys $1 \mathrm{~A}-\mathrm{Tg}$ mouse brain}

As dysbindin-1 is involved in transcriptional regulation $[28,35]$, we performed gene expression profiling in the prefrontal cortex, hippocampus, and striatum of Dys1A$\mathrm{Tg}$ mice, using the Affymetrix GeneChip. Significantly different expression between Dys1A-Tg and wild-type mice was detected in 13 genes (Table 2). The expression of other genes including endogenous (mouse) Dtnbp 1 did not statistically significantly differ between the two genotypes. We could not detect the transgenic gene (human) DTNBP1 because of species difference in nucleotide sequence. TRAF2 and NCK interacting kinase gene (Tnik) was significantly increased in both the prefrontal cortex and striatum of Dys1A-Tg mice. Ten out of 13 genes were decreased in Dys1A-Tg mice, with the most prominent being the immediate-early gene activity regulated cytoskeletal-associated protein $(A r c)$ and early growth response 2 (Egr 2$)$ in the prefrontal cortex $(0.29$ and 0.48 -fold vs. wild-type, respectively).

\section{Discussion}

We aimed to gain insight into the role of dysbindin-1 in psychiatric disorders. To this end, we first generated Dys1A-Tg mice expressing human DTNBP1 and then analyzed their phenotypes. In order to investigate the function of dysbindin-1 relevant to clinical application, human DTNBP1 was chosen as a transgene. Dys1A-Tg mice were born viable in the expected Mendelian ratios, apparently normal and fertile. Primary screening of behavior and function using the SHIRPA protocol showed a marginal change in limb grasping in Dys1A-Tg mice. They also exhibited increased hyperlocomotion after 

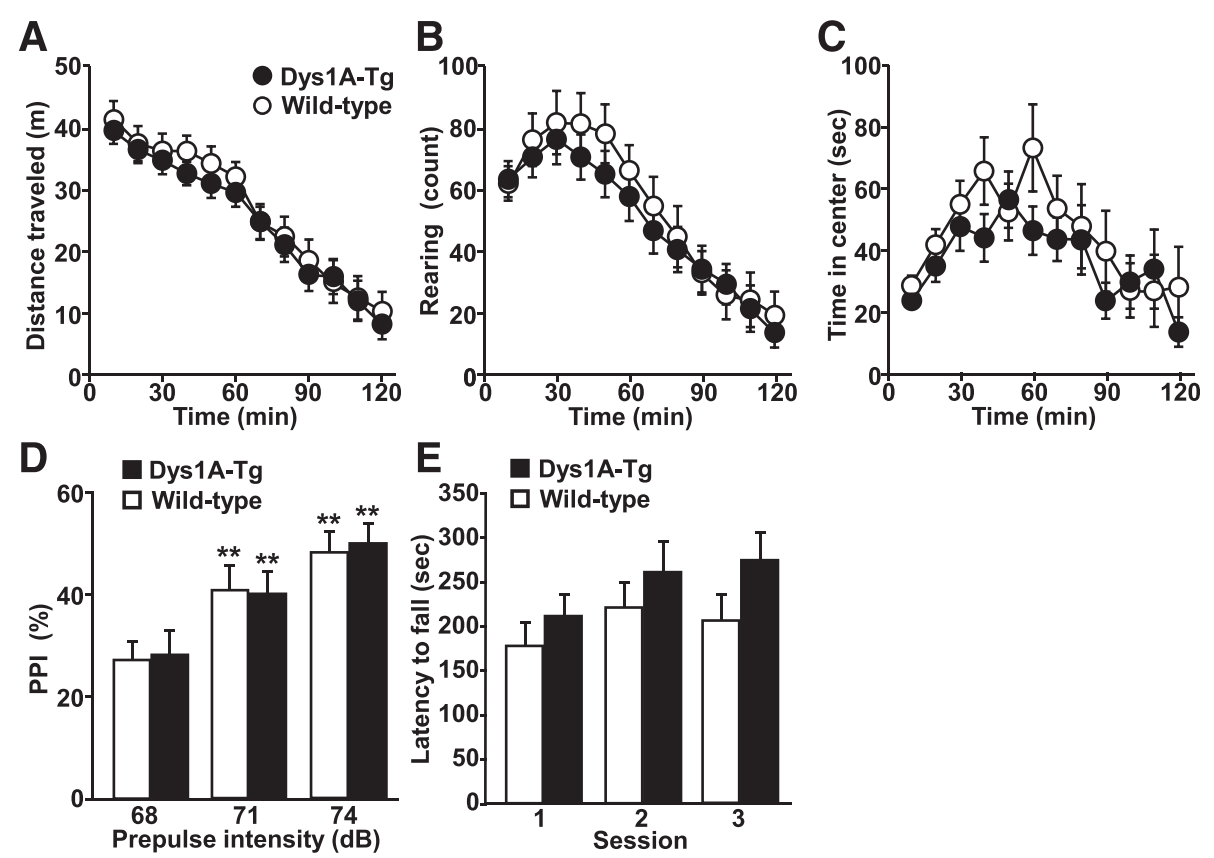

Figure 2 Essentially normal behavior in Dys1A-Tg mice under basal conditions. Distance traveled (A), vertical rearing activity (B), and time spent in the center area (C) of the open-field test, PPI of the acoustic startle response (D), and latency to fall in the accelerated rotarod test (E) were analyzed in Dys $1 \mathrm{~A}-\mathrm{Tg}$ (closed symbols and bars) and wild-type (open symbols and bars) mice. Data are expressed as mean \pm SEM. Number of mice for each genotype, 19-21 (A-C), 17-19 (D), and 17 (E). Statistical analysis was performed by repeated two-way ANOVA. ${ }^{* *} P<0.01$ vs. PPI value at $68 \mathrm{db}$ of pre-pulse intensity. There was no significant main effect of genotype in any experiment.
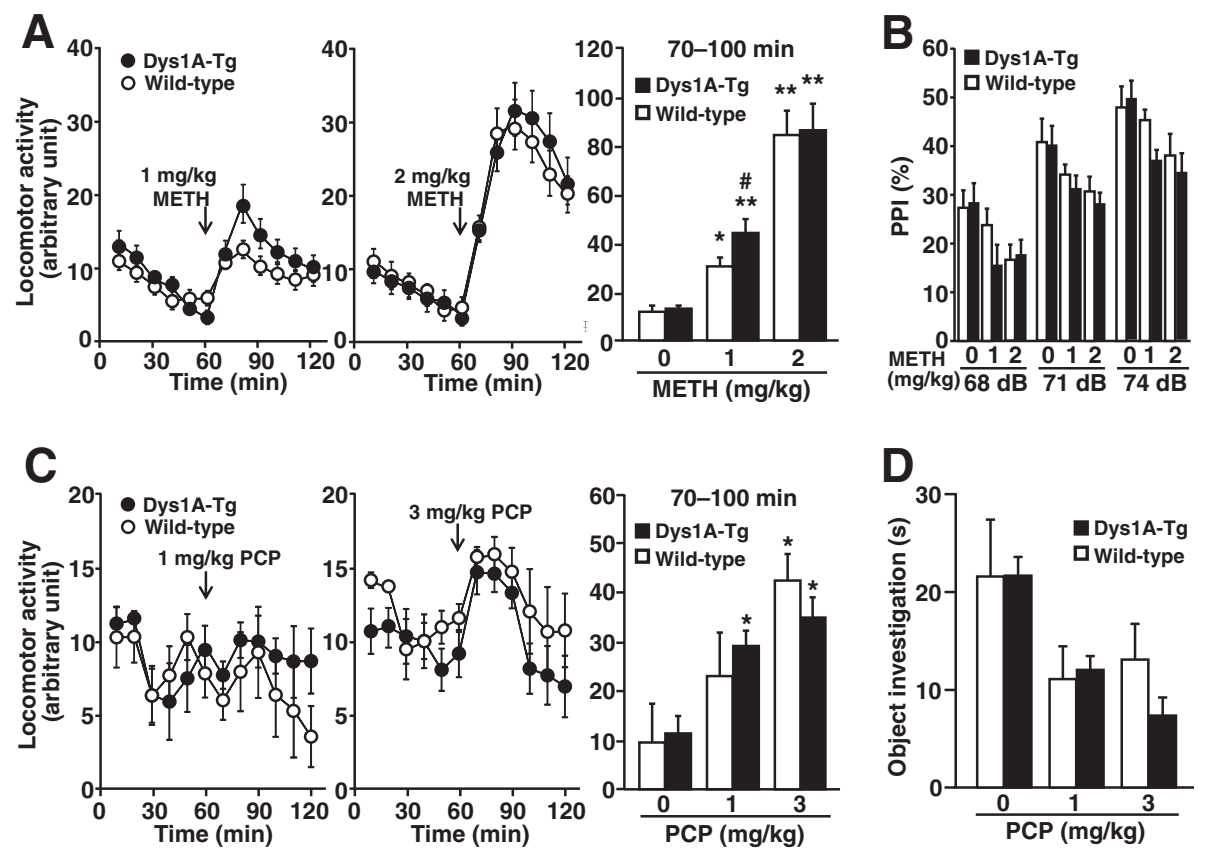

Figure 3 Slightly increased responses to METH and PCP in Dys1A-Tg mice. Acute behavioral responses to METH (A and B) or PCP (C and D) were examined using locomotor (A and C), PPI (B), and object investigation (D) tests in Dys1 A-Tg (closed symbols and bars) and wild-type (open symbols and bars) mice. METH or PCP were injected at 60 min and cumulative locomotor activity measured for 70-100 min was indicated in bar graphs in (A) and (C). Data are expressed as mean \pm SEM. Number of mice for each genotype, 14-27 (A), 17-35 (B), 3-8 (C), and 3-5 (D). Statistical analysis was performed by repeated three or two-way ANOVA followed by the Tukey-Kramer post-hoc test. ${ }^{*} P<0.05,{ }^{*} P<0.01$ vs. vehicle of the same genotype. ${ }^{\#} P<0.05$ vs. wild-type of the same treatment. 

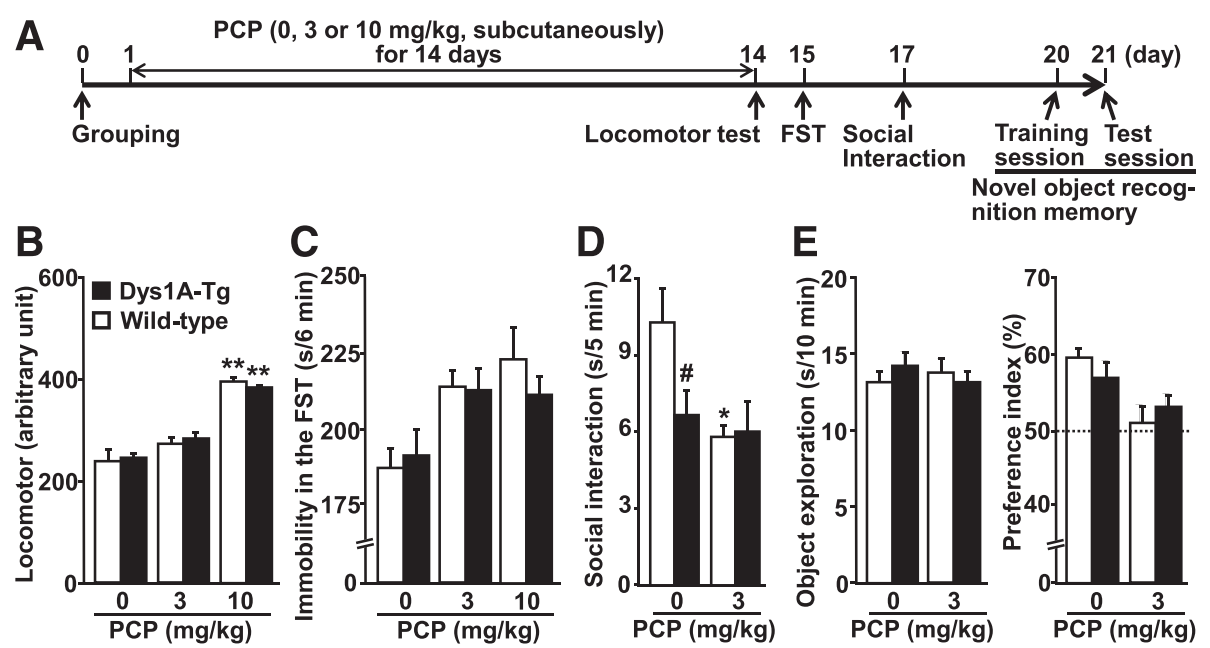

Figure 4 Effects of chronic PCP administration in Dys1A-Tg mice. (A) The experimental schedule consisted of four successive behavioral tests (see Methods for details). (B-E) PCP was administered subcutaneously daily for 14 days, and locomotor activity measured using a digital counter system with an infrared sensor for 90 min (B), immobility time in the FST (C), duration of social interaction between two unfamiliar test mice of the same genotype and treatment (D), and object exploration time and preference index in the test session of the novel object recognition memory test on day 21 (E) were determined in Dys1A-Tg (closed bars) and wild-type (open bars) mice. Data are expressed as mean \pm SEM Number of mice for each genotype is 12-14 obtained from three independent cohorts. Statistical analysis was performed by two-way ANOVA followed by the Tukey-Kramer post hoc test. ${ }^{*} P<0.05$ vs. vehicle of the same genotype, ${ }^{\#} P<0.05$ vs. wild-type of the same treatment.

Table 2 Genes with significantly altered expression in the brain of Dys1A-Tg mice

\begin{tabular}{|c|c|c|c|c|c|c|}
\hline \multirow{2}{*}{$\begin{array}{l}\text { Brain region/ } \\
\text { changes in } \\
\text { Dys } 1 \mathrm{~A}-\mathrm{Tg}\end{array}$} & \multirow[t]{2}{*}{ Gene name } & \multirow[t]{2}{*}{ Gene symbol } & \multirow[t]{2}{*}{ UniGene ID } & \multirow{2}{*}{$\begin{array}{l}\text { Fold change } \\
\text { vs. wild-type }\end{array}$} & \multicolumn{2}{|c|}{ Signal intensity } \\
\hline & & & & & Wild-type & Dys1A-Tg \\
\hline \multicolumn{7}{|c|}{ Prefrontal cortex } \\
\hline Increased & TRAF2 and NCK interacting kinase & Tnik & Mm.126193 & $1.3 \pm 0.1$ & $3765 \pm 169$ & $4742 \pm 195$ \\
\hline \multirow[t]{7}{*}{ Decreased } & Kruppel-like factor 10 & Klf10 & Mm.4292 & $0.6 \pm 0.0$ & $1055 \pm 21$ & $621 \pm 31$ \\
\hline & $\begin{array}{l}\text { Activity regulated cytoskeletal-associated } \\
\text { protein }\end{array}$ & Arc & Mm.25405 & $0.3 \pm 0.1$ & $2194 \pm 442$ & $644 \pm 156$ \\
\hline & early growth response 2 & Egr2 & Mm.290421 & $0.5 \pm 0.1$ & $633 \pm 76$ & $307 \pm 86$ \\
\hline & RIKEN cDNA 5330406 M23 gene & 5330406M23Rik & Mm.109877 & $0.7 \pm 0.0$ & $2301 \pm 117$ & $1502 \pm 86$ \\
\hline & Myelin transcription factor 1-like & Myt1l & Mm.253067 & $0.6 \pm 0.0$ & $1926 \pm 154$ & $1224 \pm 77$ \\
\hline & RIKEN cDNA C130075A20 gene & C130075A20Rik & -- & $0.5 \pm 0.0$ & $643 \pm 71$ & $348 \pm 21$ \\
\hline & Integrin beta 1 binding protein 1 & |tgb1bp1 & Mm.352231 & $0.5 \pm 0.0$ & $343 \pm 23$ & $172 \pm 14$ \\
\hline \multicolumn{7}{|l|}{ Hippocampus } \\
\hline \multirow[t]{2}{*}{ Increased } & G protein-coupled receptor 178 & Gpr178 & Mm.297552 & $1.4 \pm 0.1$ & $1512 \pm 71$ & $2169 \pm 76$ \\
\hline & Chemokine (C-C motif) ligand 21b & $\mathrm{Ccl} 21 \mathrm{~b}$ & Mm.220853 & $1.7 \pm 0.1$ & $874 \pm 78$ & $1503 \pm 65$ \\
\hline Decreased & zinc finger, MYM domain containing 1 & Zmym1 & Mm.273806 & $0.7 \pm 0.0$ & $2050 \pm 64$ & $1398 \pm 75$ \\
\hline \multicolumn{7}{|l|}{ Striatum } \\
\hline Increased & TRAF2 and NCK interacting kinase & Tnik & Mm.126193 & $1.3 \pm 0.1$ & $3418 \pm 220$ & $4587 \pm 232$ \\
\hline \multirow[t]{2}{*}{ Decreased } & $\begin{array}{l}\text { DNA segment, Chr 4, Wayne State University 53, } \\
\text { expressed }\end{array}$ & D4Wsu53e & Mm.331964 & $0.7 \pm 0.1$ & $2878 \pm 230$ & $1863 \pm 165$ \\
\hline & Kruppel-like factor 2 (lung) & Klf2 & Mm.26938 & $0.6 \pm 0.1$ & $414 \pm 62$ & $244 \pm 27$ \\
\hline
\end{tabular}


METH administration. In the brain of Dys1A-Tg mice, transcriptomic analysis identified several up- and downregulated genes, including the immediate-early genes Arc and Egr2.

Among two lines of Dys1A-Tg mice generated, total levels of dysbindin-1 (human and mouse dysbindin-1) were considered to be significantly higher in line 1 while those in line 2 were only slightly higher than wild-type mice (Figure 1C). Therefore, we performed the following behavioral and gene expression experiments in line 1. However, we could not rule out the possibility that the obtained results in the present study might be attributed to the disruption of other genes where the transgene was inserted.

In the social interaction test, which was conducted on day 17 in our test battery, we observed impairments in Dys1A-Tg mice that received vehicle (saline) for 14 days compared with wild-type mice (Figure 4D). Although PCP significantly decreased social interaction in wildtype mice, it did not further decrease the behavior in Dys1A-Tg mice. Interpretation of these results is difficult but may be related to detrimental effect in Dys1A-Tg mice caused by repeated vehicle administration for 14 days. As our previous study showed that the FST lasts for only 3 days after the last PCP injection in C57BL6/J mice [34], and it is necessary to minimize test interactions [36,37], we designed the present behavioral test battery. However, since we did not use a washout period following chronic PCP treatment, the possibility for residual acute effects of PCP may not be excluded especially in the locomotor test and the FST conducted on day 14 and day 15 , respectively, and in the mice treated with $\mathrm{PCP}$ at $10 \mathrm{mg} / \mathrm{kg}$.

As discussed above, there is a growing body of evidence implicating dysbindin-1 in psychiatric disorders [9-11,13-20], nevertheless it remains unclear how dysbindin-1 increases susceptibility to these disorders [24-26]. Dysbindin-1-deficient mutant Sandy mice (spontaneous mutant in a DBA/2 J mouse strain) display a variety of behavioral abnormalities relevant to symptoms of schizophrenia $[9,18-20]$, as well as reduced dopamine transmission in the forebrain [18]. sdy/B6 mice (Sandy mutant mice on a C57BL/6 J background) show schizophrenia-like behaviors including hyperactivity, learning and memory deficits, and disruption of dopamine/D2-related mechanisms that regulate cortical function and neuronal excitability [21,22]. These mice also exhibit increased impulsive and compulsive behaviors relevant to psychiatric disorders [23]. The present observations that Dys1A-Tg mice are essentially normal under basal conditions (except for increased limb grasping behavior) and exhibit altered behavioral responses to $\mathrm{METH}$, indicate that dysbindin-1 overexpression does not cause strong detrimental effects under basal conditions but may induce vulnerability toward psychotomimetics. The fact that either overexpression (Dys1A-Tg) or underexpression (Sandy both on DBA/2 J and C57BL/6 J backgrounds) of dysbindin-1 leads to behavioral alterations in mice highlights the functional importance of this protein and the molecular networks in which dysbindin-1 is involved.

Dysbindin-1 is expressed ubiquitously in the body and brain [1], and has been postulated to be implicated not only in psychiatric disorders such as schizophrenia, bipolar disorder, and METH psychosis [5,10-12,15-17], but also peripheral diseases such as type 7 HermanskyPudlak syndrome, which is accompanied with oculocutaneous albinism, prolonged bleeding, and pulmonary fibrosis due to abnormal vesicle trafficking to lysosomes and related organelles [2]. Thus, Dys1A-Tg mice may serve as a model for various diseases and complement dysbindin-1-null Sandy mice.

$D T N B P 1$ variants (including e.g., protective and risk haplotypes) are reported to affect susceptibility to substanceinduced psychosis [12], and dysbindin-1 is involved in regulation of synaptic plasticity $[6,9]$, neurotransmitter release [3-5], and membrane surface expression of NMDA and D2 receptors [6-8]. Altogether, it is suggested that dysbindin-1 plays significant roles in neurobehavioral control and psychiatric disorders.

\section{Conclusions}

In summary, we have generated Dys1A-Tg mice expressing human DTNBP1. Dys1A-Tg mice are apparently normal and fertile without abnormalities in their coat color, but with a marginal change in limb grasping, slightly exaggerated behavioral response to acutely administered METH. In the brain of Dys1A-Tg mice, expression levels of several genes are altered, including the immediateearly genes, Arc and Egr2. Our results in Dys1A-Tg mice further suggest a critical role for dysbindin-1 in psychiatric disorders.

\section{Methods}

\section{Ethics statement}

All animal care and handling procedures were performed according to the Guidelines for the Care and Use of Laboratory Animals approved by the Japanese Pharmacological Society, and were approved by the Animal Care and Use Committee of the Graduate School of Pharmaceutical Sciences, Osaka University. All efforts were made to minimize the number of animals used.

\section{Generation of Dys1A-Tg mice expressing human dysbindin-1}

hDTNBP1-GFP consists of Homo sapiens dysbindin-1 isoform A (accession no. NP_115498, 351 amino acids), green fluorescent protein (GFP), and a termination codon. A 1,773-bp fragment encoding 591 amino acids 
of hDTNBP1-GFP was inserted into the HapI site of the pCA-pA vector containing the CA promoter [29], and the transgene construct confirmed by DNA sequencing. Next, a 3.7-kb fragment including hDTNBP1-GFP cDNA was excised by BamHI-XhoI digestion, and used to generate Dys1A-Tg mice by pronuclear injections into fertilized C57BL/6 mouse eggs.

Genotypes were determined by PCR using genomic DNA extracted from tail biopsies in extraction buffer (5 mM EDTA, $100 \mathrm{mM}$ Tris-HCl, pH 8.5, $200 \mathrm{mM}$ $\mathrm{NaCl}, 0.2 \% \mathrm{SDS}$, and $200 \mu \mathrm{g} / \mathrm{mL}$ proteinase $\mathrm{K})$. Genotyping was performed on genomic DNA (40 ng) using AmpliTaqGold DNA Polymerase (Applied Biosystems, Foster City, CA, USA) and the following primers located in different exons of the human DTNBP1 gene $\left(5^{\prime}\right.$-GAC TAA GAA TCC ATG ACA GCA AAT C-3' and 5' -TTA ATT CTG AGG GAT TTG GAA CCT-3'; product size, $547 \mathrm{bp}$ ). The PCR reaction consisted of 40 cycles of denaturation at $94^{\circ} \mathrm{C}$ for $30 \mathrm{~s}$, annealing at $55^{\circ} \mathrm{C}$ for $30 \mathrm{~s}$, and elongation at $72^{\circ} \mathrm{C}$ for $1 \mathrm{~min}$.

Dys1A-Tg mice were backcrossed with female C57BL/6 J mice (Charles River, Osaka, Japan) for at least 10 generations. Wild-type female mice were mated with male Dys1A-Tg mice and 8-20-week-old male offspring were used for experiments. Mice were group housed under a 12-h light-dark cycle (lights on at 8:30 a.m.) with free access to food and water.

\section{RT-PCR and western blot analyses}

RT-PCR was performed as described previously, but with some modifications [38]. Briefly, total RNA was reverse transcribed and cDNA from three mice mixed and subjected to semi-quantitative RT-PCR analysis using Gotaq Hot Start Green Master Mix (Promega, Tokyo, Japan) and the following primers corresponding to different exons of the mouse Dtnbp 1 gene (5'-GAA CCA TTT GCT GCA CCT GGA C-3' and 5'-GGC CTT CTG TGT GTG CTC TGT ATC G-3'; product size, 157 bp), the human DTNBP1 gene (5'-GCA GCT CCC AGC TTT AAT CGC AG-3' and 5'-TGG GCG TGC TCT GCA TCT AGT-3'; product size, $232 \mathrm{bp}$ ), and the mouse GAPDH gene, which served as an internal control (5' -GTG TTC CCT ACC CCC AAT GTG-3' and 5'-TAC CAG GAA ATG AGC TTG AC-3'; product size, $241 \mathrm{bp}$ ). To confirm validity of genomic DNA amplification, the mouse Dtnbpl gene was amplified using intron 6-specific primers (5'-GCA CTC AGG AGA CCA TGA CA-3' and 5'-GGT TGA CAC TCT TGC GGA AT-3'; product size, 305 bp). Quantitative realtime RT-PCR was also performed in the same way as mentioned above.

Western blot analysis was performed as described $[39,40]$ using mouse monoclonal anti-dysbindin antibody, which was produced in our laboratory against glutatione S-transferase-fused human dysbindin-1 [28]. Briefly, $20 \mu \mathrm{g}$ of protein from precipitated brain homogenates were separated on SDS-PAGE and electrotransferred onto Immobilon-P Transfer Membranes (Millipore, Billerica, MA, USA), and then probed with primary antibodies: mouse monoclonal anti-dysbindin antibody (1:1,000; Cell Signaling Technology, Danvers, MA, USA) and mouse anti-GAPDH antibody (1:10,000; Millipore), followed by anti-mouse horseradish peroxidase-conjugated antibody (1:2,000; GE Healthcare, Piscataway, NJ, USA). The intensity of the bands was quantitated with Image $J$ software (National Institutes of Health, MD, USA).

\section{Drugs and experimental design for behavioral analyses}

Drug solutions were administered to mice in a volume of $0.1 \mathrm{~mL} / 10 \mathrm{~g}$ body weight. METH and PCP dissolved in saline were acutely injected intraperitoneally or subcutaneously, respectively. Each behavioral study was performed using separate cohorts of mice, except for the novel object investigation test.

In the chronic PCP administration model [34], 6week-old mice were chronically administered with PCP for 14 consecutive days, and then subjected to a battery of four different behavioral tests: locomotor analysis on day 14 (30 min after the last PCP dose), FST on day 15, social interaction test on day 17 , and novel object recognition test on days 20 (training session) and 21 (retention test session). The sequence of this behavioral test battery was fundamentally designed to minimize test interactions, by arranging the least stressful tasks first and more stressful tasks last [36,37], with the exception that the FST was performed the day after the last PCP injection as it has been shown that chronic PCP-increased immobility in the FST lasts for only 3 days in C57BL/6 J mice [34].

\section{Initial behavioral screening}

Fundamental sensory and physical functions of mice were evaluated using SHIRPA [30], with slight modifications as described previously [31].

\section{Locomotor analysis}

The open-field test was performed using the infrared Actimeter system (Panlab, Barcelona, Spain), and distance traveled, vertical rearing activity, and time spent in the center area were measured using Acti-Track software (Panlab), as described previously [41,42]. In the PCP study, locomotor activity was measured using a digital counter system with an infrared sensor (Supermex; Muromachi Kikai Co., Tokyo, Japan), as described previously [43].

\section{PPI of the acoustic startle response}

PPI of the acoustic startle response was measured in a startle chamber (SR-LAB; San Diego Instruments, San 
Diego, CA, USA), essentially as described previously [44]. PPI was calculated as percentage score for each pre-pulse trial type using the following equation: prepulse inhibition $(\%)=[1-($ startle response for pulse with pre-pulse)/(startle response for pulse alone $)] \times 100$.

\section{Rotarod test}

An accelerating rotarod treadmill (Acceler Rota-Rod 7650; Ugo Basile, Varese, Italy) was used to evaluate motor coordination and learning. Mice were first trained repeatedly at a fixed speed $(12 \mathrm{rpm})$ until the mice were able to stay on the rod for at least $300 \mathrm{~s}$. One day after training, performance on the accelerating (12-30 rpm) rotarod was examined for a maximum recording time of $600 \mathrm{~s}$. Tests were performed once for 3 consecutive days.

\section{Novel object investigation test}

Exploratory behavior towards a novel object was evaluated as described [45]. After $15 \mathrm{~min}$ habituation under dim light (40 lx) in an observation cage $(28 \mathrm{~cm}$ length $\times$ $20 \mathrm{~cm}$ width $\times 12 \mathrm{~cm}$ height), mice were presented with a novel object (a wooden ball; diameter $5 \mathrm{~cm}$ ), which was placed in the center of the cage. Duration of object exploratory behavior (sniffing or licking the wooden ball) was measured for $5 \mathrm{~min}$ from recordings by trained blinded observers. The test was performed just after locomotor analysis in the same mice treated with acute PCP.

\section{FST}

The FST was performed as described previously [46]. Briefly, behavior of mice in a glass cylinder $(19 \mathrm{~cm}$ diameter $\times 25 \mathrm{~cm}$ height) containing water $\left(25 \pm 1^{\circ} \mathrm{C}\right)$ to a depth of $13 \mathrm{~cm}$ was videotaped for $6 \mathrm{~min}$, and duration of immobility (making only minimal movements to keep floating) was measured by trained blinded observers. After the test, mice were dried thoroughly with a towel and returned to their home cage.

\section{Social interaction test}

In chronic PCP-treated mice, social interaction between adult mice was evaluated as described [33], with slight modifications. Mice were individually habituated to the observation apparatus $(35 \mathrm{~cm}$ length $\times 25 \mathrm{~cm}$ width $\times$ $25 \mathrm{~cm}$ height) for $10 \mathrm{~min}$ for 2 consecutive days. Next, two unfamiliar test mice of the same genotype and treatment were placed in the apparatus, and social interaction behavior videotaped for $5 \mathrm{~min}$. Time spent in active social interaction such as sniffing and following the partner, mounting, and crawling under/over the partner was measured by trained blinded observers.

\section{Novel object recognition memory test}

Novel object recognition memory was evaluated as described [33,34], with slight modifications [47]. Mice were individually habituated to the observation box $(30 \mathrm{~cm}$ length $\times 20 \mathrm{~cm}$ width $\times 20 \mathrm{~cm}$ height) for $10 \mathrm{~min}$ for 3 consecutive days. Next, a training session was performed, and mice were allowed to explore the observation box containing two different objects for $10 \mathrm{~min}$. After $24 \mathrm{~h}$, the retention test session was conducted, and each mouse was placed back in the observation box with a familiar object (presented in the training session) and a novel object. Behavior of the mice was videotaped and evaluated by trained blinded observers. Preference indices were calculated as the ratio of time spent exploring the novel object vs. the total time spent exploring both familiar and novel objects, and used as a dependent measure of recognition memory.

\section{Microarray analysis}

Prefrontal cortex, hippocampus, and striatum were manually dissected from the brains of nine each Dys1A-Tg and wild-type mice. Three samples were pooled and subjected to GeneChip mouse genome 4302.0 arrays (Affymetrix, Tokyo, Japan) which is one of the most comprehensive whole mouse genome expression array. A total of 18 hybridization experiments were performed according to the manufacturer's instructions, and data analyzed using GeneChip Operating Software (GCOS) v1.1.1. GCOS was used to calculate the signal intensity and percent present calls on hybridized chips. Fold change of individual genes between Dys1A-Tg and wild-type mice are presented as the ratio of normalized gene expression values in Dys1A$\mathrm{Tg}$ vs. wild-type mice.

\section{Statistical analyses}

Statistical analysis was performed using StatView (SAS Institute Japan Ltd., Tokyo, Japan). Significant differences were determined by the Student's $t$-test, MannWhitney $U$ test, $\chi^{2}$ test or two- or three-way, factorial or repeated-measures ANOVA with genotype, drug, and time as factors of variation. Tukey-Kramer post-hoc tests were also performed after significant main effects for genotype, drug, or interaction between genotype $\times$ drug were observed. The threshold for statistical significance was defined as $P<0.05$.

\section{Competing interests}

The authors declare that they have no competing interests.

\section{Authors' contributions}

RHas, TK, TNab, MT, and HH conceived the study. NS, RHas, HT, AM, TM, SM, TK, TNab, MT, and HH designed the experiments. NS, RHas, and HH wrote the manuscript. NS, YO, HT, TNag, SU, AM, TM, RHab, and YA performed the behavioral experiments, and NS, YO, HT, YA and $\mathrm{HH}$ analyzed the data. RHas, HT, SU, SM, TK, HY, and YY generated Dys1A-Tg mice. RHas, SU, SM, TK, HY, TNak, and KN performed the gene expression analysis and analyzed the data. All authors read and approved the final manuscript. 


\section{Acknowledgments}

We are grateful to Dr. Takayoshi Inoue at the National Center of Neurology and Psychiatry for help and advice on constructing the hDTNBP1-GFP construct. This work was supported in part by the Japan Society for the Promotion of Science Grants-in-Aid for Scientific Research, KAKENHI (Grant Nos. 23790086 (NS); 22390225, 23659565, and 25293250 (RHas); 26293020 and $26670122(\mathrm{HH})$ ), Research Fellowships for Young Scientists (YO), the Funding Program for Next Generation World-Leading Researchers (Grant No. LS081 (HH)), and grants for research from the Uehara Memorial Foundation, Japan $(\mathrm{HH})$.

\section{Author details}

'Laboratory of Molecular Neuropharmacology, Graduate School of Pharmaceutical Sciences, Osaka University, 1-6 Yamadaoka, Suita, Osaka 565-0871, Japan. ${ }^{2}$ Molecular Research Center for Children's Mental Development, United Graduate School of Child Development, Osaka University, Kanazawa University, Hamamatsu University School of Medicine, Chiba University and University of Fukui, 2-2 Yamadaoka, Suita, Osaka 565-0871, Japan. ${ }^{3}$ Department of Psychiatry, Graduate School of Medicine, Osaka University, 2-2 Yamadaoka, Suita, Osaka 565-0871, Japan. ${ }^{4}$ Department of Molecular Neuropsychiatry, Graduate School of Medicine, Osaka University, 2-2 Yamadaoka, Suita, Osaka 565-0871, Japan. ${ }^{5}$ Department of Chemical Pharmacology, Graduate School of Pharmaceutical Sciences, Meijo University, 150 Yagotoyama, Tempaku-ku, Nagoya 468-8503, Japan. ${ }^{6}$ Molecular Brain Science, United Graduate School of Child Development, Osaka University, Kanazawa University, Hamamatsu University School of Medicine, Chiba University and University of Fukui, 2-2 Yamadaoka, Suita, Osaka 565-0871, Japan. ${ }^{7}$ Anatomy and Neuroscience, Graduate School of Medicine, Osaka University, 2-2 Yamadaoka, Suita, Osaka 565-0871, Japan. ${ }^{8}$ iPS Cell-based Research Project on Brain Neuropharmacology and Toxicology, Graduate School of Pharmaceutical Sciences, Osaka University, 1-6 Yamadaoka, Suita, Osaka 565-0871, Japan. ' ${ }^{2}$ aboratory of Medicinal Pharmacology, Graduate School of Pharmaceutical Sciences, Osaka University, 1-6 Yamadaoka, Suita, Osaka 565-0871, Japan. ${ }^{10}$ Department of Mental Disorder Research, National Institute of Neuroscience, National Center of Neurology and Psychiatry, 4-1-1 Ogawahigashicho, Kodaira, Tokyo 187-8502, Japan. " Department of Regional Pharmaceutical Care \& Sciences, Graduate School of Pharmaceutical Sciences, Meijo University, 150 Yagotoyama, Tenpaku-ku, Nagoya 468-8503, Japan.

\section{Received: 6 August 2014 Accepted: 25 September 2014}

Published online: 09 October 2014

\section{References}

1. Benson MA, Newey SE, Martin-Rendon E, Hawkes R, Blake DJ: Dysbindin, a novel coiled-coil-containing protein that interacts with the dystrobrevins in muscle and brain. J Biol Chem 2001, 276:24232-24241.

2. Li W, Zhang Q, Oiso N, Novak EK, Gautam R, O'Brien EP, Tinsley CL, Blake DJ, Spritz RA, Copeland NG, Jenkins NA, Amato D, Roe BA, Starcevic M, Dell'Angelica EC, Elliott RW, Mishra V, Kingsmore SF, Paylor RE, Swank RT: Hermansky-Pudlak syndrome type 7 (HPS-7) results from mutant dysbindin, a member of the biogenesis of lysosome-related organelles complex 1 (BLOC-1). Nat Genet 2003, 35:84-89.

3. Chen XW, Feng YQ, Hao CJ, Guo XL, He X, Zhou ZY, Guo N, Huang HP, Xiong W, Zheng H, Zuo PL, Zhang CX, Li W, Zhou Z: DTNBP1, a schizophrenia susceptibility gene, affects kinetics of transmitter release. J Cell Biol 2008, 181:791-801.

4. Jentsch JD, Trantham-Davidson H, Jairl C, Tinsley M, Cannon TD, Lavin A: Dysbindin modulates prefrontal cortical glutamatergic circuits and working memory function in mice. Neuropsychopharmacology 2009, 34:2601-2608.

5. Numakawa T, Yagasaki Y, Ishimoto T, Okada T, Suzuki T, Iwata N, Ozaki N, Taguchi T, Tatsumi M, Kamijima K, Straub RE, Weinberger DR, Kunugi H, Hashimoto R: Evidence of novel neuronal functions of dysbindin, a susceptibility gene for schizophrenia. Hum Mol Genet 2004, 13:2699-2708.

6. Tang TT, Yang F, Chen BS, Lu Y, Ji Y, Roche KW, Lu B: Dysbindin regulates hippocampal LTP by controlling NMDA receptor surface expression. Proc Natl Acad Sci U S A 2009, 106:21395-21400.

7. lizuka Y, Sei Y, Weinberger DR, Straub RE: Evidence that the BLOC-1 protein dysbindin modulates dopamine D2 receptor internalization and signaling but not D1 internalization. J Neurosci 2007, 27:12390-12395.

8. Ji Y, Yang F, Papaleo F, Wang HX, Gao WJ, Weinberger DR, Lu B: Role of dysbindin in dopamine receptor trafficking and cortical GABA function. Proc Natl Acad Sci U S A 2009, 106:19593-19598.
9. Glen WB Jr, Horowitz B, Carlson GC, Cannon TD, Talbot K, Jentsch JD, Lavin A: Dysbindin-1 loss compromises NMDAR-dependent synaptic plasticity and contextual fear conditioning. Hippocampus 2014, 24:204-213.

10. Straub RE, Jiang Y, MacLean CJ, Ma Y, Webb BT, Myakishev MV, Harris-Kerr C, Wormley B, Sadek H, Kadambi B, Cesare AJ, Gibberman A, Wang X, O'Neill FA, Walsh D, Kendler KS: Genetic variation in the 6p22.3 gene DTNBP1, the human ortholog of the mouse dysbindin gene, is associated with schizophrenia. Am J Hum Genet 2002, 71:337-348.

11. Breen G, Prata D, Osborne S, Munro J, Sinclair M, Li T, Staddon S, Dempster D, Sainz R, Arroyo B, Kerwin RW, St Clair D, Collier D: Association of the dysbindin gene with bipolar affective disorder. Am J Psychiatry 2006, 163:1636-1638.

12. Kishimoto M, Ujike H, Motohashi Y, Tanaka Y, Okahisa Y, Kotaka T, Harano M, Inada T, Yamada M, Komiyama T, Hori T, Sekine Y, Iwata N, Sora I, Iyo M, Ozaki N, Kuroda S: The dysbindin gene (DTNBP1) is associated with methamphetamine psychosis. Biol Psychiatry 2008, 63:191-196.

13. Hashimoto R, Noguchi H, Hori H, Nakabayashi T, Suzuki T, Iwata N, Ozaki N, Kosuga A, Tatsumi M, Kamijima K, Harada S, Takeda M, Saitoh O, Kunugi H: A genetic variation in the dysbindin gene (DTNBP1) is associated with memory performance in healthy controls. World J Biol Psychiatry 2010, 11:431-438.

14. Hashimoto R, Noguchi H, Hori H, Ohi K, Yasuda Y, Takeda M, Kunugi H: Association between the dysbindin gene (DTNBP1) and cognitive functions in Japanese subjects. Psychiatry Clin Neurosci 2009, 63:550-556.

15. Weickert CS, Straub RE, McClintock BW, Matsumoto M, Hashimoto R, Hyde TM, Herman MM, Weinberger DR, Kleinman JE: Human dysbindin (DTNBP1) gene expression in normal brain and in schizophrenic prefrontal cortex and midbrain. Arch Gen Psychiatry 2004, 61:544-555.

16. Bray NJ, Preece A, Williams NM, Moskvina V, Buckland PR, Owen MJ, O'Donovan MC: Haplotypes at the dystrobrevin binding protein 1 (DTNBP1) gene locus mediate risk for schizophrenia through reduced DTNBP1 expression. Hum Mol Genet 2005, 14:1947-1954.

17. Talbot K, Eidem WL, Tinsley CL, Benson MA, Thompson EW, Smith RJ, Hahn CG, Siegel SJ, Trojanowski JQ, Gur RE, Blake DJ, Arnold SE: Dysbindin-1 is reduced in intrinsic, glutamatergic terminals of the hippocampal formation in schizophrenia. J Clin Invest 2004, 113:1353-1363.

18. Hattori S, Murotani T, Matsuzaki S, Ishizuka T, Kumamoto N, Takeda M, Tohyama M, Yamatodani A, Kunugi H, Hashimoto R: Behavioral abnormalities and dopamine reductions in sdy mutant mice with a deletion in Dtnbp1, a susceptibility gene for schizophrenia. Biochem Biophys Res Commun 2008, 373:298-302.

19. Feng YQ, Zhou ZY, He X, Wang H, Guo XL, Hao CJ, Guo Y, Zhen XC, Li W: Dysbindin deficiency in sandy mice causes reduction of snapin and displays behaviors related to schizophrenia. Schizophr Res 2008, 106:218-228.

20. Takao K, Toyama K, Nakanishi K, Hattori S, Takamura H, Takeda M, Miyakawa T, Hashimoto R: Impaired long-term memory retention and working memory in sdy mutant mice with a deletion in Dtnbp1, a susceptibility gene for schizophrenia. Mol Brain 2008, 1:11.

21. Cox MM, Tucker AM, Tang J, Talbot K, Richer DC, Yeh L, Arnold SE: Neurobehavioral abnormalities in the dysbindin-1 mutant, sandy, on a C57BL/6 J genetic background. Genes Brain Behav 2009, 8:390-397.

22. Papaleo F, Yang F, Garcia S, Chen J, Lu B, Crawley JN, Weinberger DR: Dysbindin-1 modulates prefrontal cortical activity and schizophrenia-like behaviors via dopamine/D2 pathways. Mol Psychiatry 2012, 17:85-98.

23. Carr GV, Jenkins KA, Weinberger DR, Papaleo F: Loss of dysbindin-1 in mice impairs reward-based operant learning by increasing impulsive and compulsive behavior. Behav Brain Res 2013, 241:173-184.

24. Strohmaier J, Frank J, Wendland JR, Schumacher J, Jamra RA, Treutlein J, Nieratschker V, Breuer R, Mattheisen M, Herms S, Muhleisen TW, Maier W, Nothen MM, Cichon S, Rietschel M, Schulze TG: A reappraisal of the association between Dysbindin (DTNBP1) and schizophrenia in a large combined case-control and family-based sample of German ancestry. Schizophr Res 2010, 118:98-105.

25. Balu DT, Coyle JT: Neuroplasticity signaling pathways linked to the pathophysiology of schizophrenia. Neurosci Biobehav Rev 2011, 35:848-870

26. Yamamori H, Hashimoto R, Verrall L, Yasuda Y, Ohi K, Fukumoto M, UmedaYano S, Ito A, Takeda M: Dysbindin-1 and NRG-1 gene expression in immortalized lymphocytes from patients with schizophrenia. J Hum Genet 2011, 56:478-483. 
27. Papaleo F, Burdick MC, Callicott JH, Weinberger DR: Epistatic interaction between COMT and DTNBP1 modulates prefrontal function in mice and in humans. Mol Psychiatry 2014, 19:311-316.

28. Okuda H, Kuwahara R, Matsuzaki S, Miyata S, Kumamoto N, Hattori T, Shimizu S, Yamada K, Kawamoto K, Hashimoto R, Takeda M, Katayama T, Tohyama M: Dysbindin regulates the transcriptional level of myristoylated alanine-rich protein kinase $C$ substrate via the interaction with NF-YB in mice brain. PLoS One 2010, 5:e8773.

29. Niwa H, Yamamura K, Miyazaki J: Efficient selection for high-expression transfectants with a novel eukaryotic vector. Gene 1991, 108:193-199.

30. Rogers DC, Fisher EM, Brown SD, Peters J, Hunter AJ, Martin JE: Behavioral and functional analysis of mouse phenotype: SHIRPA, a proposed protocol for comprehensive phenotype assessment. Mamm Genome 1997, 8:711-713

31. Kato T, Kasai A, Mizuno M, Fengyi L, Shintani N, Maeda S, Yokoyama M, Ozaki M, Nawa H: Phenotypic characterization of transgenic mice overexpressing neuregulin-1. PLOS One 2010, 5:e14185.

32. Arai S, Takuma K, Mizoguchi H, Ibi D, Nagai T, Takahashi K, Kamei H, Nabeshima T, Yamada K: Involvement of pallidotegmental neurons in methamphetamine- and MK-801-induced impairment of prepulse inhibition of the acoustic startle reflex in mice: reversal by GABAB receptor agonist baclofen. Neuropsychopharmacology 2008, 33:3164-3175.

33. Hida H, Mouri A, Ando Y, Mori K, Mamiya T, Iwamoto K, Ozaki N, Yamada K Nabeshima T, Noda Y: Combination of neonatal Polyl:C and adolescent phencyclidine treatments is required to induce behavioral abnormalities with overexpression of GLAST in adult mice. Behav Brain Res 2014, 258:34-42.

34. Mouri A, Koseki T, Narusawa S, Niwa M, Mamiya T, Kano S, Sawa A, Nabeshima T: Mouse strain differences in phencyclidine-induced behavioural changes. Int I Neuropsychopharmacol 2012, 15:767-779.

35. Ma X, Fei E, Fu C, Ren H, Wang G: Dysbindin-1, a schizophrenia-related protein, facilitates neurite outgrowth by promoting the transcriptional activity of p53. Mol Psychiatry 2011, 16:1105-1116.

36. Takao K, Yamasaki N, Miyakawa T: Impact of brain-behavior phenotypying of genetically-engineered mice on research of neuropsychiatric disorders. Neurosci Res 2007, 58:124-132.

37. Crawley JN: Behavioral phenotyping strategies for mutant mice. Neuron 2008, 57:809-818.

38. Mabuchi T, Shintani N, Matsumura S, Okuda-Ashitaka E, Hashimoto H, Muratani T, Minami T, Baba A, Ito S: Pituitary adenylate cyclase-activating polypeptide is required for the development of spinal sensitization and induction of neuropathic pain. J Neurosci 2004, 24:7283-7291.

39. Kubota K, Kumamoto N, Matsuzaki S, Hashimoto R, Hattori T, Okuda H, Takamura H, Takeda M, Katayama T, Tohyama M: Dysbindin engages in c-Jun N-terminal kinase activity and cytoskeletal organization. Biochem Biophys Res Commun 2009, 379:191-195.

40. Nihonmatsu-Kikuchi N, Hashimoto R, Hattori S, Matsuzaki S, Shinozaki T, Miura H, Ohota S, Tohyama M, Takeda M, Tatebayashi Y: Reduced rate of neural differentiation in the dentate gyrus of adult dysbindin null (sandy) mouse. PLoS One 2011, 6:e15886.

41. Fujii $H$, Ishihama $T$, Ago $Y$, Shintani $N$, Kakuda M, Hashimoto $H$, Baba A Matsuda T: Methamphetamine-induced hyperactivity and behavioral sensitization in PACAP deficient mice. Peptides 2007, 28:1674-1679.

42. Ishihama T, Ago Y, Shintani N, Hashimoto H, Baba A, Takuma K, Matsuda T: Environmental factors during early developmental period influence psychobehavioral abnormalities in adult PACAP-deficient mice. Behav Brain Res 2010, 209:274-280.

43. Hashimoto H, Shintani N, Tanaka K, Mori W, Hirose M, Matsuda T, Sakaue M, Miyazaki J, Niwa H, Tashiro F, Yamamoto K, Koga K, Tomimoto S, Kunugi A Suetake S, Baba A: Altered psychomotor behaviors in mice lacking pituitary adenylate cyclase-activating polypeptide (PACAP). Proc Natl Acad Sci U S A 2001, 98:13355-13360.

44. Hazama K, Hayata-Takano A, Uetsuki K, Kasai A, Encho N, Shintani N, Nagayasu K, Hashimoto R, Reglodi D, Miyakawa T, Nakazawa T, Baba A, Hashimoto H: Increased behavioral and neuronal responses to a hallucinogenic drug in PACAP heterozygous mutant mice. PLoS One 2014, 9:e89153.

45. Haba R, Shintani N, Onaka Y, Kanoh T, Wang H, Takenaga R, Hayata A, Hirai H, Nagata KY, Nakamura M, Kasai A, Hashimoto R, Nagayasu K, Nakazawa T, Hashimoto H, Baba A: Central CRTH2, a second prostaglandin D2 receptor, mediates emotional impairment in the lipopolysaccharide and tumor-induced sickness behavior model. J Neurosci 2014, 34:2514-2523.
46. Hashimoto H, Hashimoto R, Shintani N, Tanaka K, Yamamoto A, Hatanaka M, Guo X, Morita Y, Tanida M, Nagai K, Takeda M, Baba A: Depression-like behavior in the forced swimming test in PACAP-deficient mice: amelioration by the atypical antipsychotic risperidone. J Neurochem 2009, 110:595-602.

47. Ago $Y$, Hiramatsu N, Ishihama T, Hazama K, Hayata-Takano A, Shibasaki $Y$, Shintani N, Hashimoto H, Kawasaki T, Onoe H, Chaki S, Nakazato A, Baba A, Takuma K, Matsuda T: The selective metabotropic glutamate 2/3 receptor agonist MGS0028 reverses psychomotor abnormalities and recognition memory deficits in mice lacking the pituitary adenylate cyclase-activating polypeptide. Behav Pharmacol 2013, 24:74-77.

doi:10.1186/s13041-014-0074-x

Cite this article as: Shintani et al:: Behavioral characterization of mice overexpressing human dysbindin-1. Molecular Brain 2014 7:74.

\section{Submit your next manuscript to BioMed Central and take full advantage of:}

- Convenient online submission

- Thorough peer review

- No space constraints or color figure charges

- Immediate publication on acceptance

- Inclusion in PubMed, CAS, Scopus and Google Scholar

- Research which is freely available for redistribution

Submit your manuscript at www.biomedcentral.com/submit
C) Biomed Central 